\title{
Nomogram for predicting axillary lymph node status after neoadjuvant chemotherapy in breast cancer
}

\author{
Mengshen Wang ${ }^{1 \#} \wedge$, Mozhi Wang ${ }^{1 *} \wedge$, Zhenning Wang ${ }^{2}$, Yongxi Song ${ }^{2}$, Peng Gao ${ }^{2}$, Pengliang Wang ${ }^{2}$, \\ Chong Wang ${ }^{1}$, Xueting Yu ${ }^{1}$, Fengheng $\mathrm{Wei}^{1}$, Jingyi Guo ${ }^{1}$, Yingying $\mathrm{Xu}^{1}{ }^{\wedge}$ \\ ${ }^{1}$ Department of Breast Surgery, the First Affiliated Hospital of China Medical University, Shenyang, China; ${ }^{2}$ Department of Surgical Oncology, the \\ First Hospital of China Medical University, Shenyang, China \\ Contributions: (I) Conception and design: Y Xu; (II) Administrative support: Y Xu, M Wang, M Wang; (III) Provision of study materials or patients: \\ M Wang, M Wang; (IV) Collection and assembly of data: X Yu, F Wei, J Guo, M Wang, M Wang; (V) Data analysis and interpretation: Z Wang, Y \\ Song, P Wang, C Wang; (VI) Manuscript writing: All authors; (VII) Final approval of manuscript: All authors. \\ \#These authors contributed equally to this work. \\ Correspondence to: Yingying Xu. Department of Breast Surgery, the First Affiliated Hospital of China Medical University, 155 North Nanjing Street, \\ Shenyang, China. Email: xuyingying@cmu.edu.cn.
}

Background: Many breast cancer patients benefit from neoadjuvant chemotherapy (NAC). However, sentinel lymph node biopsy (SLNB) after NAC remains controversial, especially for patients with axillary lymph node metastasis (ALNM) at diagnosis. We developed a nomogram for predicting axillary lymph node (ALN) status after NAC to screen for patients for whom SLNB may be beneficial.

Methods: A total of 320 cT1-4N0-1M0 breast cancer patients receiving ALN dissection (ALND) after NAC were included. Univariate and multivariate logistic regression analyses determined significant factors for predicting ALN status. Efficacy of the resulting nomogram was assessed using receiver operating characteristic (ROC) and calibration curves, while decision curve analysis (DCA) was used to evaluated net clinical benefit. Our nomogram was validated using female patients grouped according to a diagnosis of node-positive ( $\mathrm{cN} 1)$ or node-negative ( $\mathrm{cN} 0)$ by ultrasound-guided needle biopsy of suspected lymph nodes before NAC.

Results: Logistic regression analyses indicated that estrogen receptor (ER), Ki67, degree of tumor regression, clinical tumor T stage after NAC, and ALN Breast Imagining-Reporting and Data System (BI-RADS) category after NAC, were associated with ALN status. The resulting nomogram had an area under the curve (AUC) of 0.802 [95\% confidence interval (CI), 0.7485-0.8554], and the calibration plot showed strong uniformity between predicted and actual ALN status. DCA indicated a positive net benefit of nomogram predictions in our cohort. After internal validation, the $\mathrm{cN} 1$ and $\mathrm{cN} 0$ groups had an AUC of 0.7926 (95\% CI, 0.7187-0.8665) and 0.8165 (95\% CI, 0.7381-0.8949), respectively. The calibration plot indicated better performance in the $\mathrm{cN} 0$ group.

Conclusions: After NAC, some patients may benefit from SLNB. Our nomogram predicts ALN status after NAC and has great potential to assist in clinical decision-making.

Keywords: Breast cancer; neoadjuvant chemotherapy (NAC); nomogram; sentinel lymph node biopsy (SLNB)

Submitted Jun 21, 2020. Accepted for publication Sep 18, 2020.

doi: $10.21037 /$ tcr-20-2377

View this article at: http://dx.doi.org/10.21037/tcr-20-2377

^ ORCID: Yingying Xu, 0000-0003-4924-9379; Mengshen Wang, 0000-0002-3190-6062; Mozhi Wang, 0000-0002-0297-2052. 


\section{Introduction}

Breast cancer is the most common malignant tumor among females, and the leading cause of tumor-related death worldwide (1,2). In 2017 in the United States, 252,710 females were diagnosed with breast cancer, and breast cancer caused 40,610 deaths (14.4\% of total tumor-related fatalities) (1). Neoadjuvant chemotherapy (NAC) is a treatment for patients with local advanced breast cancer. It is also useful for some early-stage breast cancer patients whose tumor size does not permit breast conservation. Several cycles of NAC can grant some patients a chance for breast-conserving surgery, and a subset of patients with axillary lymph nodes (ALNs) was reported achieving pathological complete response (pCR), indicating a good prognosis $(3,4)$. The National Surgical Adjuvant Breast and Bowel Project (NSABP) Protocols B-18 and B-27 assert that preoperative therapy is equivalent to adjuvant therapy for some patients (5). For local advanced breast cancer, mastectomy combined with ALN dissection (ALND) is a standard procedure. However, this can injury axillary lymph vessels and nerves, resulting in physical and psychological morbidities, and poor quality of life (6). The National Comprehensive Cancer Network Clinical Practice Guidelines in Oncology Version 1.2018 (NCCN Guidelines) (7) state that ALND should be done for clinically positive ALNs following NAC, while sentinel lymph node biopsy (SLNB) can be performed for clinically negative ALNs following NAC, avoiding possible ALND-related morbidities in these patients.

Until now, there has been no published nomogram that predicts ALN status after NAC in breast cancer patients regardless of their initial ALN condition. Therefore, we conducted this study to construct a nomogram to identify those patients suitable for receiving SLNB.

We present the following article in accordance with the TRIPOD reporting checklist (available at http://dx.doi. org/10.21037/tcr-20-2377).

\section{Methods}

\section{Patients}

This was a retrospective study of 320 females who received NAC for cT1-4N0-1M0 breast cancer at the First Hospital of China Medical University between 2013 and 2017. The inclusion criteria were as follows: (I) patients with invasive breast cancer confirmed by core needle biopsy before NAC; (II) patients who received at least one cycle of NAC; (III) patients with no anti-tumor treatments prior to NAC; and (IV) patients who underwent mastectomy and ALND following NAC. The exclusion criteria were as follows: (I) patients with missing data; (II) patients who suffered distant metastasis prior to surgery; or (III) patients who had surgical histories of ipsilateral axilla. The study was conducted in accordance with the Declaration of Helsinki (as revised in 2013). The study was approved by the Ethical Committee of The First Hospital of China Medical University (201972-2), and informed consent was taken from all the patients.

\section{Patbology}

Data extracted from tumor samples included expression of estrogen receptor (ER), progesterone receptor (PR), human epithelial growth receptor 2 (HER2), and Ki67. These were measured by immunohistochemistry (IHC) performed on formalin-fixed, paraffin-embedded tissue sections by the Pathology Department of The First Hospital of China Medical University, using standard protocols. ER and PR positivity were defined as nuclear staining in $>1 \%$ of tumor cells. HER2 positivity was defined as either scoring $3+$ by IHC or amplification by fluorescent in situ hybridization (FISH) (8). Ki67 was divided into two groups, Ki67<14\% and $\geq 14 \%$, with the latter considered high (9). Histology grade was assessed according to the Elston-Ellis system (10). ALN status after NAC was recorded after ALND. All samples were examined independently by at least two experienced pathologists.

All patients received breast and ALN ultrasound examinations prior to NAC, with the longest primary tumor diameter present recorded as the baseline. After the final cycle of NAC before surgery, all patients underwent another breast and ALN ultrasound examination, and the longest diameter after NAC was compared to the baseline.

\section{Neoadjuvant treatment regimens}

Patients were classified into four groups according to NAC regimens. Group 1 received epirubicin or doxorubicin but no docetaxel. Group 2 received docetaxel but no epirubicin or doxorubicin. Group 3 received epirubicin or doxorubicin and docetaxel. Group 4 contained two subgroups, HER2+ with/without Herceptin treatment. Patients received four cycles of NAC on average.

\section{Statistical analysis}

Statistical analyses for the comparisons included the 
Student's $t$-test for continuous variables and the Chi-square test or Fisher's exact test for categorical variables. A P value $<0.05$ was considered significant. Data analyses were performed using SPSS version 22.0 (SPSS Inc, Chicago, IL, USA). $\mathrm{R}$ software was used in constructing the nomogram (Institute for Statistics and Mathematics, Vienna, Austria, Version 3.4.1, www.R-project.org).

\section{Results}

\section{Description of enrolled patients}

General characteristics of the 320 enrolled patients are shown in Table 1. Median age was 51 (ranging from 24 to 76) years. Of the patients with ALN metastasis (ALNM), 159 (71.3\%) were ER positive and 64 (28.7\%) were ER negative $(\mathrm{P}=0.001)$. Average Ki67 index was significantly higher in the ALN with no metastasis (ALNNM) cohort compared with the ALNM cohort (45.5\% vs. $36.1 \%$, respectively; $\mathrm{P}=0.008)$. More than half the patients in the ALNM cohort presented as ycT2-3, while the majority of patients in the ALNNM cohort were ycT1 $(\mathrm{P}<0.001)$. For ALN category based on Breast Imaging-Reporting and Data System (BI-RADS) under ultrasound after NAC, there were 68 (30.5\%) ALNM patients and 72 (74.2\%) ALNNM patients with ALN BI-RADS category $\leq 3$, with the reverse outcome occurring for ALN BI-RADS category $\geq 4(69.5 \%$ vs. $25.8 \%, \mathrm{P}<0.001)$. Clinical tumor stage before NAC, PR status, HER2 status, NAC courses, and NAC regimens all showed no significant difference between the ALNM and ALNNM groups.

\section{Construction of the nomogram}

We constructed the nomogram based on statistically and clinically significant factors (Table 1). The following were evaluated by univariate analysis: clinical tumor stage before and after NAC (cT and ycT, respectively), patient age, ER, PR, HER2, Ki67, histological grades of core biopsy samples, NAC regimens, number of cycles, ALN BI-RADS category before and after NAC, and tumor regression degree (ratio between longest diameter of the primary tumor before and after NAC measured by ultrasound, with the cutoff value set at 1.0 such that a fold change $>1$ indicates that the primary tumor size is decreasing, and $\leq 1$ indicates that the primary tumor has a limited response to the chemotherapy regimen). A multivariate logistic regression analysis was conducted on the meaningful variables, including ALN BIRADS category before NAC $(\mathrm{P}=0.002)$, histologic grade $(\mathrm{P}=0.050)$, ER ( $\mathrm{P}=0.001)$, Ki67 ( $\mathrm{P}=0.491)$, tumor regression degree $(\mathrm{P}=0.087)$, yc T $(\mathrm{P}<0.001)$, and ALN BI-RADS category after NAC $(\mathrm{P}<0.001)$. We determined that ER $(\mathrm{P}=0.009)$, yc $\mathrm{T}(\mathrm{P}=0.005)$ and ALN BI-RADS category after NAC $(\mathrm{P}<0.001)$ were significant factors. These five variables were used for the construction of our nomogram (Table 2). While primary tumor regression degree and Ki67 can reflect the response to NAC (11-14), these were not

Table 1 Clinical and pathological features of the study population

\begin{tabular}{|c|c|c|c|c|}
\hline Features & All patients, $\mathrm{n}(\%)$ & ALNM, n (\%) & ALNNM, n (\%) & $P$ value \\
\hline Total no. of patients & 320 & 223 & 97 & \\
\hline Age & & & & 0.940 \\
\hline Mean (SD) & 50.8 & $51.6(9.7)$ & $48.9(9.8)$ & \\
\hline Range & $24-76$ & $26-76$ & $24-66$ & \\
\hline Clinical tumor stage before NAC (CT) & & & & 0.517 \\
\hline cT1-2 & $233(72.8)$ & $160(71.7)$ & $73(75.3)$ & \\
\hline cT3-4 & $87(27.2)$ & $63(28.3)$ & $24(24.7)$ & \\
\hline ALN BI-RADS category before NAC & & & & 0.002 \\
\hline$\leq 3$ & $44(13.8)$ & $22(9.9)$ & $22(22.7)$ & \\
\hline$\geq 4$ & $276(86.2)$ & $201(90.1)$ & $75(77.3)$ & \\
\hline Histologic grade & & & & 0.050 \\
\hline
\end{tabular}

Table 1 (contiuned) 
Table 1 (contiuned)

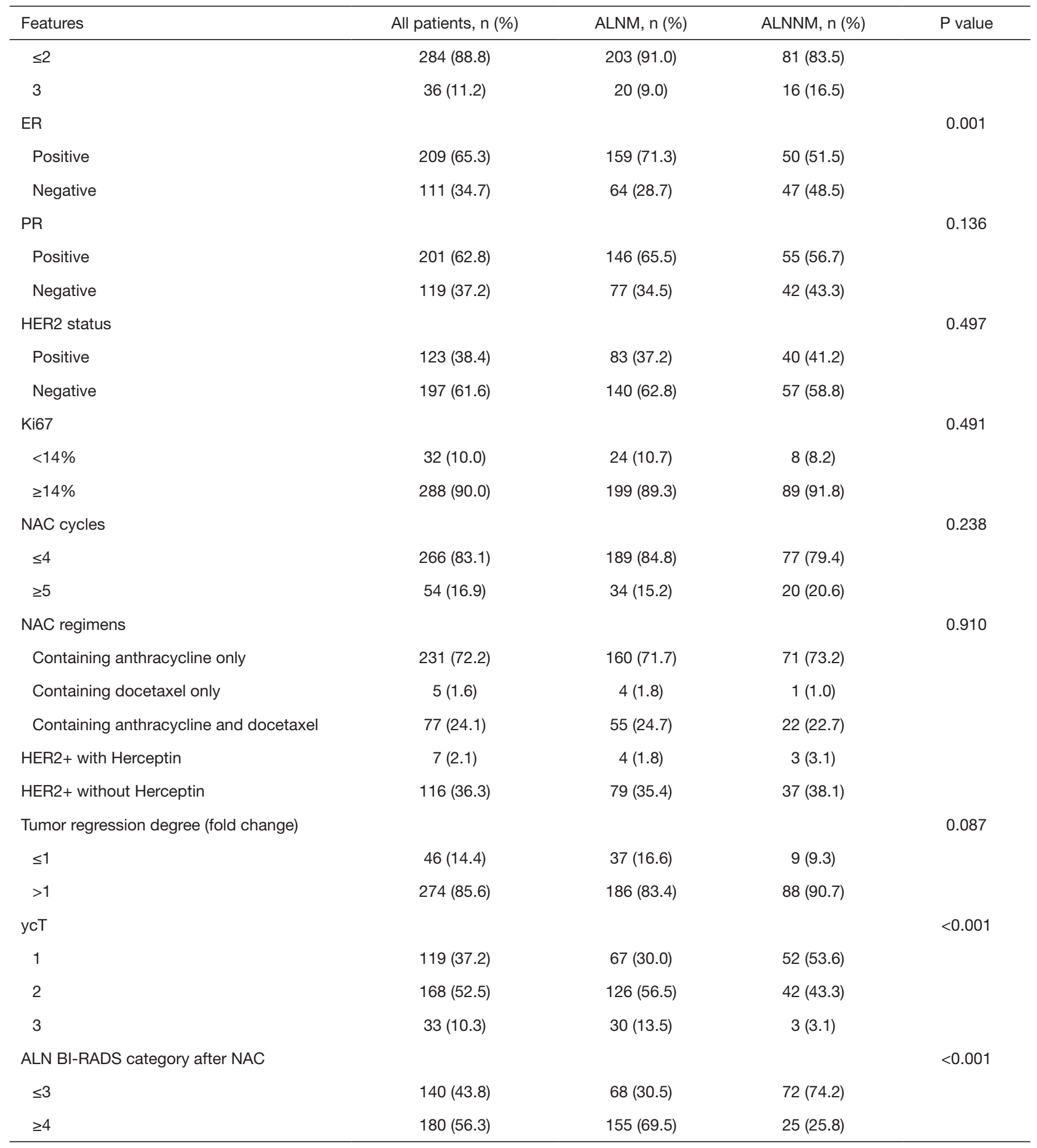

ALNM, axillary lymph node metastasis; ALNNM, axillary lymph node with no metastasis; NAC, neoadjuvant chemotherapy; ALN, axillary lymph node; BI-RADS, Breast Imagining-Reporting and Data System; ER, estrogen receptor; PR, progesterone receptor; HER2, human epidermal growth factor receptor 2; ycT, clinical tumor T stage after NAC; ALN BI-RADS category before NAC, ALN category based on BI-RADS under ultrasound before NAC; ALN BI-RADS category after NAC, ALN category based on BI-RADS under ultrasound after NAC. 
Table 2 Multivariate analysis of ALNM factors of breast cancer patients after receiving NAC

\begin{tabular}{|c|c|c|c|}
\hline Variables & OR & $95 \% \mathrm{Cl}$ & $P$ value \\
\hline \multicolumn{3}{|c|}{ ALN BI-RADS category before NAC } & 0.216 \\
\hline$\leq 3$ & - & - & \\
\hline$\geq 4$ & 0.613 & $0.283-1.330$ & \\
\hline \multicolumn{3}{|l|}{ Histological grade } & 0.313 \\
\hline$\leq 2$ & - & - & \\
\hline 3 & 1.585 & $0.648-3.877$ & \\
\hline \multicolumn{3}{|l|}{ ER } & 0.009 \\
\hline Negative & - & - & \\
\hline Positive & 0.427 & $0.226-0.805$ & \\
\hline Ki67 & 0.989 & $0.976-1.002$ & 0.110 \\
\hline \multicolumn{3}{|c|}{ Tumor regression degree (fold change) } & 0.672 \\
\hline$\leq 1$ & - & - & \\
\hline$>1$ & 1.218 & $0.490-3.026$ & \\
\hline \multicolumn{4}{|l|}{ ycT } \\
\hline 1 & - & - & \\
\hline 2 & 0.147 & $0.038-0.564$ & 0.005 \\
\hline 3 & 0.296 & $0.079-1.113$ & 0.072 \\
\hline \multicolumn{3}{|c|}{ ALN BI-RADS category after NAC } & $<0.001$ \\
\hline$\leq 3$ & - & - & \\
\hline$\geq 4$ & 0.172 & $0.094-0.315$ & \\
\hline
\end{tabular}

ALNM, axillary lymph node metastasis; NAC, neoadjuvant chemotherapy; $\mathrm{Cl}$, confidence interval; ALN, axillary lymph node; BI-RADS, Breast Imagining-Reporting and Data System; $E R$, estrogen receptor; ycT, clinical tumor T stage after NAC; ALN BI-RADS category before NAC, ALN category based on BI-RADS under ultrasound before NAC; ALN BI-RADS category after NAC, ALN category based on BI-RADS under ultrasound after NAC.

significant variables in our analysis. This may be due to the limited number of cases.

Figure 1 shows the nomogram for predicting the probability of ALN with metastasis after NAC. For each covariate, we drew a vertical line upwards from its respective axis to the "points" bar on top to calculate its individual point value. After summing all the covariate points, we drew a vertical line downwards from the "total points" line to evaluate ALNM risk. The figure clearly shows that ALN BI-RADS category after NAC makes the greatest contribution to the total score, while ER, Ki67, ycT, and tumor regression degree have moderate influences.

\section{Quantifying the performance of the nomogram}

We constructed the receiver operating characteristic (ROC) to validate the nomogram internally. The area under the curve (AUC) was 0.802 [95\% confidence interval (CI), $0.7485-0.8554]$, indicating a fairly accurate predictive ability (Figure 2).

Bootstrapping is a form of internal validation which involves repeatedly applying the model to randomly selected sample sets from the original cohort. By using a calibration plot with bootstrap sampling $(n=1,000)$, we internally calibrated the performance of the nomogram (Figure 3) and found good agreement between the bias-corrected prediction and the ideal reference line. This indicated that the nomogram performed well.

Decision curve analysis (DCA) (15) was used to determine the clinical validity of the nomogram in our cohort. Because the range of threshold probabilities were wide, the results indicated a good clinical applicability of the model in predicting the probability of ALN with metastasis after NAC (Figure 4).

The enrolled population had varying ALN status before NAC. To explore the value of the nomogram in different groups, the population was divided into two groups: $\mathrm{cN} 1$ and $\mathrm{cN} 0$. The ROC curve for the $\mathrm{cN} 1$ group was illustrated in Figure $5 \mathrm{~A}$ with an AUC of 0.7926 (95\% CI, 0.7187-0.8665) and calibration plots for the $\mathrm{cN} 1$ was shown in Figure 5B. Figure $6 A$ shows the ROC curve for the $\mathrm{cN} 0$ group, with an AUC of 0.8165 (95\% CI, 0.7381-0.8949), and Figure $6 B$ describes calibration plots for the $\mathrm{cN} 0$. These results indicate substantial predictive power of the nomogram as well as good agreement between the actual and expected outcomes.

\section{Discussion}

NAC is commonly used for breast cancer patients with local advanced cancer or patients with larger primary tumors who desire breast-conserving surgery. After several cycles of NAC, some patients experience tumor reduction with positive lymph nodes becoming negative. NAC also acts as a chemo-sensitivity test in vivo, guiding doctors in choosing appropriate therapy regimens. Patients whose tumors are unresponsive to NAC should have their chemotherapy 


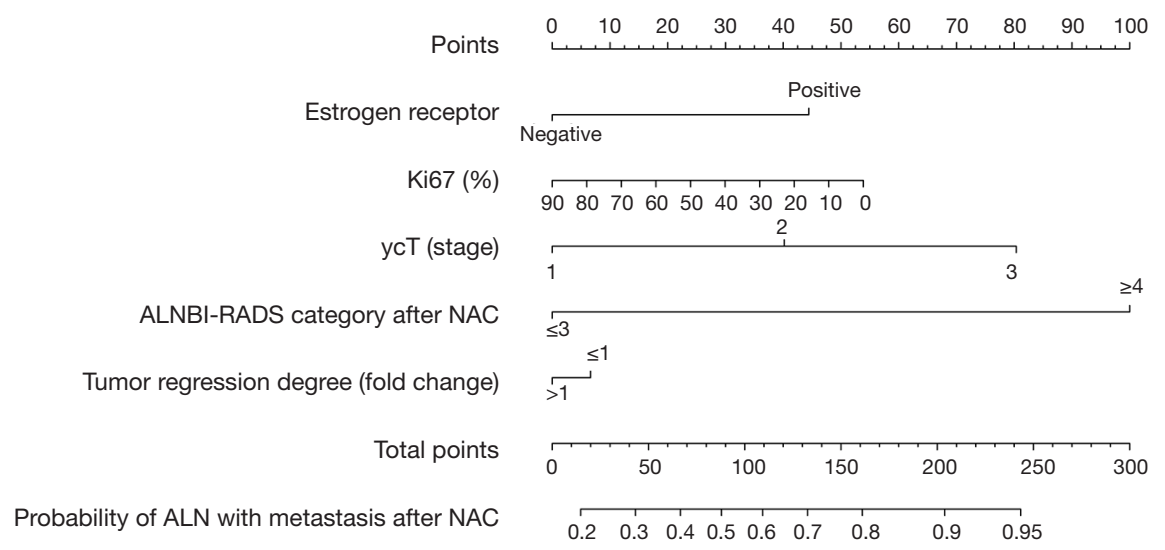

Figure 1 Nomogram for predicting the probability of ALN with metastasis after NAC. ALN, axillary lymph node; NAC, neoadjuvant chemotherapy; BI-RADS, Breast Imagining-Reporting and Data System.

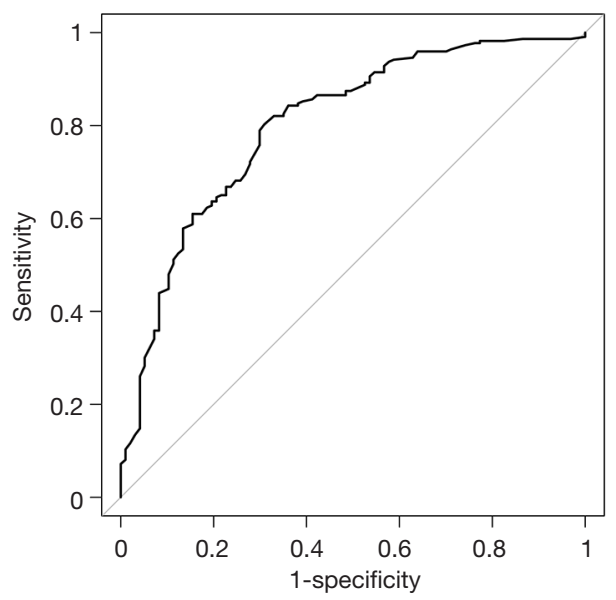

Figure 2 Discrimination plot. The AUC for the ROC was 0.802 (95\% CI, 0.7485-0.8554). AUC, area under the curve; ROC, receiver operating characteristic; $\mathrm{CI}$, confidence interval.

regimens changed.

According to the NCCN guidelines, SLNB can be performed in $\mathrm{cN} 0$ patients after neoadjuvant systemic therapy. In $\mathrm{cN} 1$ patients, axilla may be restaged after neoadjuvant systemic therapy. When ALNs are clinically positive, ALND should be performed. When ALN are clinically negative, ALND or SLNB can be performed. However, SLNB remains controversial.

Some patients with initial ALNM confirmed by core needle biopsy may progress to metastasis-free lymph nodes following NAC. A meta-analysis revealed a pCR rate of $36.8 \%$ (95\% CI, 34.2-39.5\%) (16). ALN pCR is

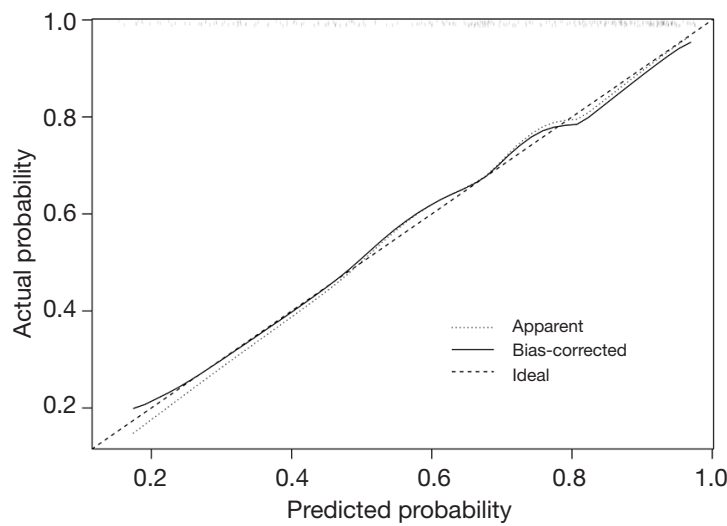

Figure 3 Calibration plot of the nomogram predicting the probability of ALN with metastasis after NAC. The X-axis and $\mathrm{Y}$-axis indicate the predicted and actual probability of ALN with metastasis after NAC, respectively. A perfect prediction would correspond to a slope of 1 . ALN, axillary lymph node; NAC, neoadjuvant chemotherapy.

a well-known predictor of disease-free survival, allowing patients to safely avoid ALND (17). The traditional surgery method is ALND, which may result in side effects such as lymphedema, upper limb numbness, arm pain, limited arm movement, and difficulty handling liquid gathering in the armpit. There is insufficient evidence to recommend SLNB for patients following NAC treatment (18), as lymphatic drainage could be disturbed, making the sentinel lymph node difficult to find. Importantly, tumor cells throughout the axilla may react to NAC differently, with some ALNs responding well and reaching pCR, and others remaining 


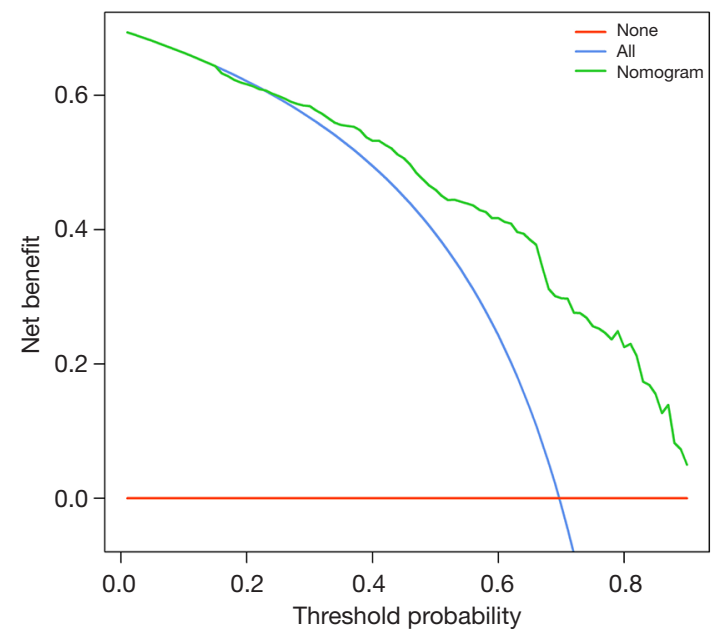

Figure 4 DCA for nomogram-predicted probabilities of ALNM in our cohort. Theoretical results for all patients with negative ALN or with positive ALN after NAC are represented by red and blue lines, respectively. The green line indicates the net benefit of using the nomogram. DCA, decision curve analysis; ALNM, axillary lymph node metastasis; ALN, axillary lymph node; NAC, neoadjuvant chemotherapy; ycT, clinical T stage after NAC.

positive for tumor cells (19-22), leading to unacceptable false-negative rates (FNRs). In several studies assessing the identification rate (IR) and FNR of SLNB after NAC, the FNR range was $9-33 \%$, while the IR range was $86.7-100 \%$ (23-27). In a meta-analysis, IR range was $78-95 \%$, while the FNR range was $5-25 \%$ for breast cancer patients with clinically positive nodes after NAC (19). Although some authors have suggested the use of clipping the nodes at the point of initial biopsy for identification after NAC and before surgery. However, modeling with limited data to predict lymph node status after NAC may not only improve accuracy but also minimize patient suffering. In view of the high pCR rate of lymph nodes after NAC, and the inevitable FNR of SLNB, we combined clinical pathological factors and other predictors into a nomogram to evaluate the status of ALN after NAC, and then filtered a group of patients receiving SLNB to show its confidence.

We developed a nomogram based on the clinical and pathological parameters of primary tumors to evaluate ALN status in breast cancer patients following NAC treatment. Tumor characteristics of 320 patients who underwent NAC were analyzed. Only ER, ycT, and ALN BI-RADS category after NAC were identified as independent predictors of ALNM through multivariate logistic regression analysis.
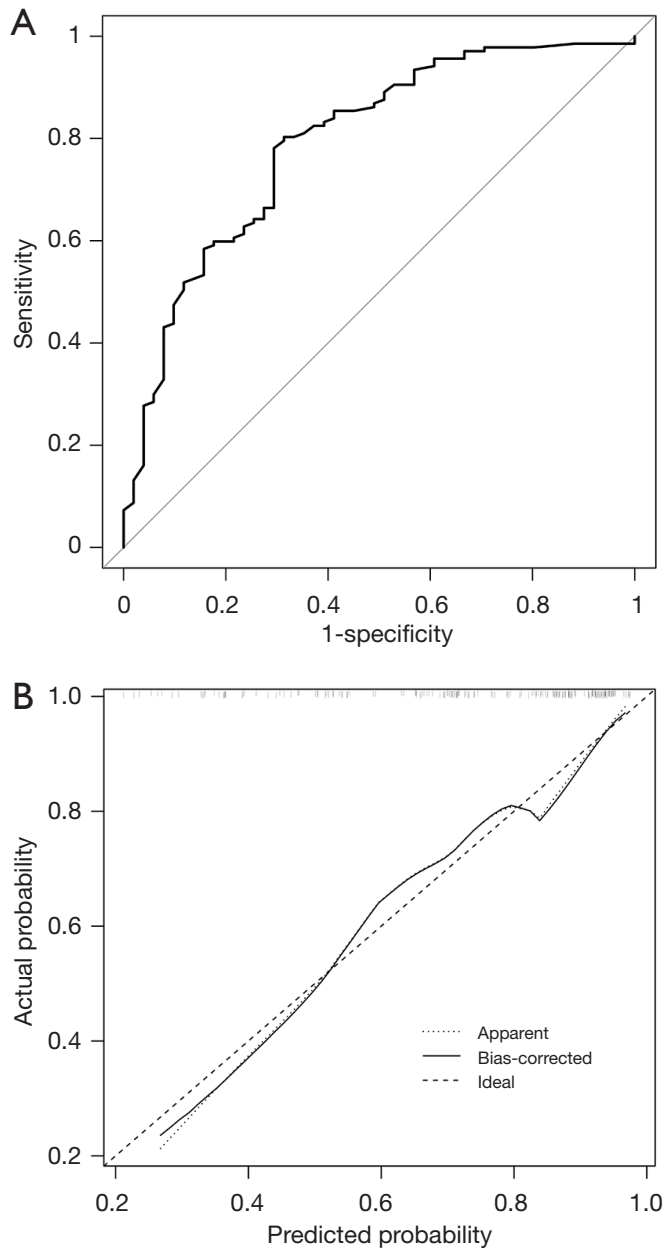

Figure 5 Validation of the nomogram in the cN1 group. (A) Internal validation in the $\mathrm{cN} 1$ group. The AUC of the ROC was 0.7926 (95\% CI, 0.7187-0.8665); (B) calibration plot in the $\mathrm{cN} 1$ group. AUC, area under the curve; ROC, receiver operating characteristic; CI, confidence interval.

Interestingly, Ki67 was a meaningful factor in univariate analysis, but not after multivariate analysis. Tumor regression degree of primary tumors and Ki67 reflect the response to NAC (11-14); therefore, we included these in our nomogram. The resulting nomogram showed a discriminative ability for AUC of 0.802 (95\% CI, $0.7485-$ 0.8554). DCA also showed a favorable predictive profile for evaluating ALN status after NAC, providing a more precise and individualized prediction.

Several previous nomograms have been developed for breast cancer patients receiving NAC (28-31), including ER, PR, HER2, Ki67, age, histology grade, initial clinical 

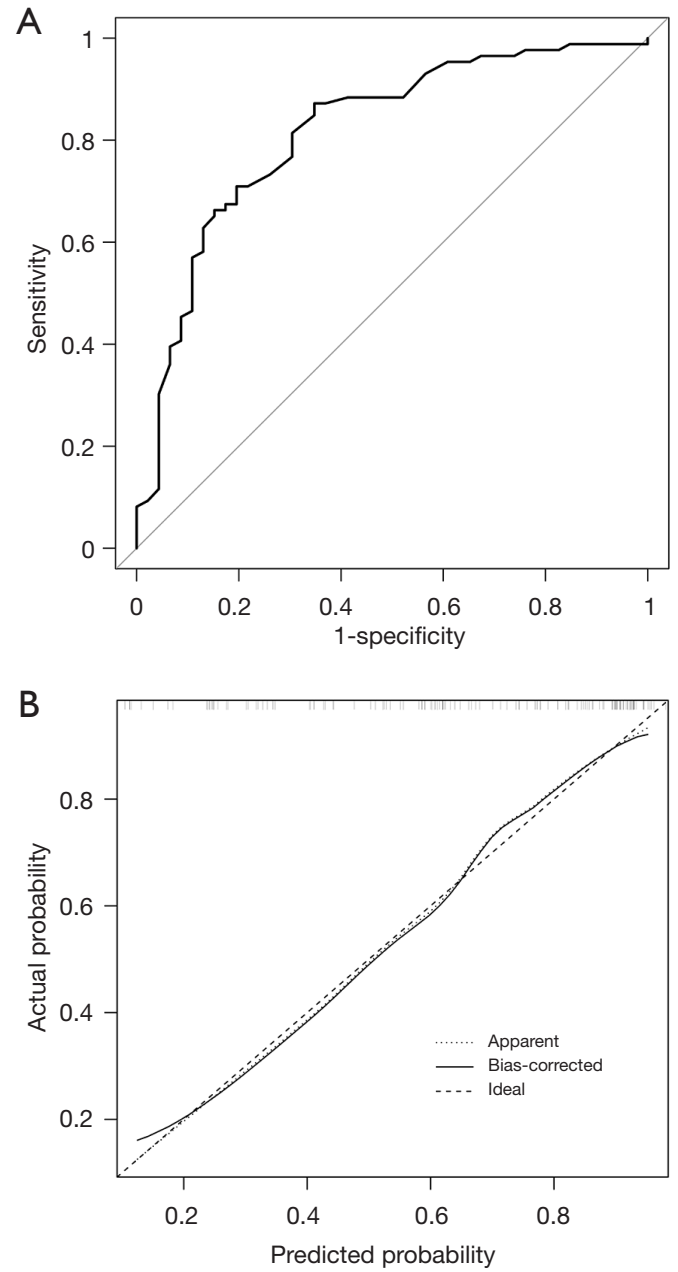

Figure 6 Validation of the nomogram in the cN0 group. (A) Internal validation in the $\mathrm{cN} 0$ group. The AUC for the ROC was 0.8165 (95\% CI, 0.7381-0.8949); (B) calibration plot in the cN0 group. AUC, area under the curve; ROC, receiver operating characteristic; CI, confidence interval.

T stage, and number of courses as covariates. For our nomogram, we considered ER and Ki67, as well as the less frequently considered yc T, tumor regression degree, and ALN BI-RADS category after NAC. In our statistical analysis, yc T $(\mathrm{P}=0.005)$ and ALN BI-RADS category after NAC $(\mathrm{P}<0.001)$ showed strong significance, and our nomogram had an AUC of 0.802, which is higher than most existing models. Additionally, the subgroup internal validation of our model in both the $\mathrm{cN} 1$ group and $\mathrm{cN} 0$ group showed good results.

Despite the good performance of our model, additional steps are needed to enhance the predictive ability. According to the NCCN guidelines, the FNR for SLNB is reduced by using dual tracer or by removing two or more sentinel nodes for patients confirmed to be node-positive before NAC (7). Previous studies have subdivided their population into two groups, comparing those with only one sentinel lymph node removed versus those patients with two or more sentinel lymph nodes removed during the operation (32-35). Subgroup analysis showed that the FNR was significantly improved if two or more sentinel nodes were removed versus only one, with a pooled FNR of $10.4 \%$ and $23.9 \%$, respectively $(\mathrm{P}=0.026)(16)$. After enrolling 756 patients who received NAC, Boughey et al. reported that mapping techniques were the only factors influencing SLN identification. By comparing blue dye alone with blue dye plus radio-labelled colloid, the SLN IR improved from $78.6 \%$ to $93.8 \%(\mathrm{P}=0.006)$, indicating that optimal tracer use is important for enhancing the IR (36). Our nomogram could be used in addition to this, by classifying patients into several groups with different probabilities of ALNM. For patients suitable for SLNB, future work should focus on screening for patients with different risks of ALNM to receive diverse tracer agents.

There are several limitations in our study. First, it was a single-center analysis, and validation by an external database may improve the model's performance. We searched several existing public databases but were unable to find a suitable database that included all our target predictors (ER, ycT, ALN BI-RADS category after NAC, Ki67, tumor regression degree). Second, due to the limited data of patients treated with NAC in the single institution, there may be other significant factors related to ALNM that were not included in our nomogram. Third, for HER2+ patients in our cohort, only a small portion received antiHER2-therapy. Whether NAC regimen affects the status of ALN after NAC is worthy of further discussion, and more patients who received anti-HER2-therapy should be included in future studies. Finally, obtaining all our data from a single institution might have led to selection bias. Consequently, we need to assemble a multicenter database of patient data to optimize and validate the nomogram in the future.

\section{Conclusions}

In conclusion, the present study identified five predictors to construct a nomogram which predicts ALN status after receiving NAC, regardless of the initial status of ALN in 
breast cancer patients. This may assist in determining the best option for patients in relation to axillary surgery after NAC.

\section{Acknowledgments}

Funding: This work was supported by the National Natural Science Foundation of China (81773083), the Scientific and Technological Innovation Leading Talent Project of Liaoning Province (XLYC1802108), and the Support Project for Young and Technological Innovation Talents of Shenyang (RC190393).

\section{Footnote}

Reporting Checklist: The authors have completed the TRIPOD reporting checklist. Available at http://dx.doi. org/10.21037/tcr-20-2377

Data Sharing Statement: Available at http://dx.doi. org/10.21037/tcr-20-2377

Peer Review File: Available at http://dx.doi.org/10.21037/tcr20-2377

Conflicts of Interest: All authors have completed the ICMJE uniform disclosure form (available at http://dx.doi. org/10.21037/tcr-20-2377). The authors have no conflicts of interest to declare.

Ethical Statement: The authors are accountable for all aspects of the work in ensuring that questions related to the accuracy or integrity of any part of the work are appropriately investigated and resolved. The study was conducted in accordance with the Declaration of Helsinki (as revised in 2013). The study was approved by the Ethical Committee of The First Hospital of China Medical University (2019-72-2), and informed consent was taken from all the patients.

Open Access Statement: This is an Open Access article distributed in accordance with the Creative Commons Attribution-NonCommercial-NoDerivs 4.0 International License (CC BY-NC-ND 4.0), which permits the noncommercial replication and distribution of the article with the strict proviso that no changes or edits are made and the original work is properly cited (including links to both the formal publication through the relevant DOI and the license).
See: https://creativecommons.org/licenses/by-nc-nd/4.0/.

\section{References}

1. Siegel RL, Miller KD, Jemal A. Cancer statistics, 2017. CA Cancer J Clin 2017;67:7-30.

2. Chen $W$, Zheng R, Baade $P$, et al. Cancer statistics in China, 2015. CA Cancer J Clin 2016;66:115-32.

3. Hennessy BT, Hortobagyi GN, Rouzier R, et al. Outcome after pathologic complete eradication of cytologically proven breast cancer axillary node metastases following primary chemotherapy. J Clin Oncol 2005;23:9304-11.

4. Rouzier R, Extra J, Klijanienko J, et al. Incidence and prognostic significance of complete axillary downstaging after primary chemotherapy in breast cancer patients with $\mathrm{T} 1$ to $\mathrm{T} 3$ tumors and cytologically proven axillary metastatic lymph nodes. J Clin Oncol 2002;20:1304-10.

5. Rastogi $\mathrm{P}$, Anderson S, Bear H, et al. Preoperative chemotherapy: updates of National Surgical Adjuvant Breast and Bowel Project Protocols B-18 and B-27. J Clin Oncol 2008;26:778-85.

6. Fleissig A, Fallowfield L, Langridge C, et al. Postoperative arm morbidity and quality of life. Results of the ALMANAC randomised trial comparing sentinel node biopsy with standard axillary treatment in the management of patients with early breast cancer. Breast Cancer Res Treat 2006;95:279-93.

7. NCCN clinical practice guidelines in oncology. Breast cancer. Version 1. 2018. Available online: https://www. nccn.org/professionals/physician_gls/default.aspx

8. Wolff AC, Hammond ME, Hicks DG, et al. Recommendations for human epidermal growth factor receptor 2 testing in breast cancer: American Society of Clinical Oncology/College of American Pathologists clinical practice guideline update. Arch Pathol Lab Med 2014;138:241-56.

9. Cheang MC, Chia SK, Voduc D, et al. Ki67 index, HER2 status, and prognosis of patients with luminal B breast cancer. J Natl Cancer Inst 2009;101:736-50.

10. Elston CW, Ellis IO. Pathological prognostic factors in breast cancer. I. The value of histological grade in breast cancer: experience from a large study with long-term follow-up. Histopathology 2002;41:154-61.

11. Keam B, Im SA, Park S, et al. Nomogram predicting clinical outcomes in breast cancer patients treated with neoadjuvant chemotherapy. J Cancer Res Clin Oncol 2011;137:1301-8.

12. Sueta A, Yamamoto $Y$, Hayashi M, et al. Clinical 
significance of pretherapeutic Ki67 as a predictive parameter for response to neoadjuvant chemotherapy in breast cancer: is it equally useful across tumor subtypes? Surgery 2014;155:927-35.

13. Kurozumi S, Inoue K, Takei H, et al. ER, PgR, Ki67, p27(Kip1), and histological grade as predictors of pathological complete response in patients with HER2positive breast cancer receiving neoadjuvant chemotherapy using taxanes followed by fluorouracil, epirubicin, and cyclophosphamide concomitant with trastuzumab. BMC Cancer 2015;15:622.

14. Li XB, Krishnamurti U, Bhattarai S, et al. Biomarkers predicting pathologic complete response to neoadjuvant chemotherapy in breast cancer. Am J Clin Pathol 2016;145:871-8.

15. Vickers AJ, Elkin EB. Decision curve analysis: a novel method for evaluating prediction models. Med Decis Making 2006;26:565-74.

16. van Nijnatten TJ, Schipper RJ, Lobbes MB, et al. The diagnostic performance of sentinel lymph node biopsy in pathologically confirmed node positive breast cancer patients after neoadjuvant systemic therapy: a systematic review and meta-analysis. Eur J Surg Oncol 2015;41:1278-87.

17. Kim HS, Shin MS, Kim CJ, et al. Improved model for predicting axillary response to neoadjuvant chemotherapy in patients with clinically node-positive breast cancer. J Breast Cancer 2017;20:378-85.

18. Lyman GH, Giuliano AE, Somerfield MR, et al. American Society of Clinical Oncology guideline recommendations for sentinel lymph node biopsy in early-stage breast cancer. J Clin Oncol 2005;23:7703-20.

19. Fu JF, Chen HL, Yang J, et al. Feasibility and accuracy of sentinel lymph node biopsy in clinically node-positive breast cancer after neoadjuvant chemotherapy: a metaanalysis. PLoS One 2014;9:e105316.

20. Geng C, Chen X, Pan X, et al. The feasibility and accuracy of sentinel lymph node biopsy in initially clinically nodenegative breast cancer after neoadjuvant chemotherapy: a systematic review and meta-analysis. PLoS One 2016;11:e0162605.

21. Takahashi M, Jinno H, Hayashida T, et al. Correlation between clinical nodal status and sentinel lymph node biopsy false negative rate after neoadjuvant chemotherapy. World J Surg 2012;36:2847-52.

22. Yu Y, Cui N, Li HY, et al. Sentinel lymph node biopsy after neoadjuvant chemotherapy for breast cancer: retrospective comparative evaluation of clinically axillary lymph node positive and negative patients, including those with axillary lymph node metastases confirmed by fine needle aspiration. BMC Cancer 2016;16:808.

23. Nason KS, Anderson BO, Byrd DR, et al. Increased false negative sentinel node biopsy rates after preoperative chemotherapy for invasive breast carcinoma. Cancer 2000;89:2187-94.

24. Gimbergues P, Abrial C, Durando X, et al. Sentinel lymph node biopsy after neoadjuvant chemotherapy is accurate in breast cancer patients with a clinically negative axillary nodal status at presentation. Ann Surg Oncol 2008;15:1316-21.

25. Schwartz GF, Meltzer AJ. Accuracy of axillary sentinel lymph node biopsy following neoadjuvant (induction) chemotherapy for carcinoma of the breast. Breast J 2003;9:374-9.

26. Kinoshita T. Sentinel lymph node biopsy is feasible for breast cancer patients after neoadjuvant chemotherapy. Breast Cancer 2007;14:10-5.

27. Tafra L, Verbanac KM, Lannin DR. Preoperative chemotherapy and sentinel lymphadenectomy for breast cancer. Am J Surg 2001;182:312-5.

28. Rouzier R, Pusztai L, Delaloge S, et al. Nomograms to predict pathologic complete response and metastasis-free survival after preoperative chemotherapy for breast cancer. J Clin Oncol 2005;23:8331-9.

29. Vila J, Mittendorf E, Farante G, et al. Nomograms for predicting axillary response to neoadjuvant chemotherapy in clinically node-positive patients with breast cancer. Ann Surg Oncol 2016;23:3501-9.

30. Kim JY, Park HS, Kim S, et al. Prognostic nomogram for prediction of axillary pathologic complete response after neoadjuvant chemotherapy in cytologically proven node-positive breast cancer. Medicine (Baltimore) 2015;94:e1720.

31. Schipper RJ, Moossdorff M, Nelemans PJ, et al. A model to predict pathologic complete response of axillary lymph nodes to neoadjuvant chemo(immuno)therapy in patients with clinically node-positive breast cancer. Clin Breast Cancer 2014;14:315-22.

32. Boileau JF, Poirier B, Basik M, et al. Sentinel node biopsy after neoadjuvant chemotherapy in biopsy-proven nodepositive breast cancer: the SN FNAC study. J Clin Oncol 2015;33:258-64.

33. Boughey JC, Suman VJ, Mittendorf EA, et al. Sentinel lymph node surgery after neoadjuvant chemotherapy in patients with node-positive breast cancer: the ACOSOG Z1071 (Alliance) clinical trial. JAMA 2013;310:1455-61. 
34. Park S, Park JM, Cho JH, et al. Sentinel lymph node biopsy after neoadjuvant chemotherapy in patients with cytologically proven node-positive breast cancer at diagnosis. Ann Surg Oncol. 2013;20:2858-65.

35. Yagata H, Yamauchi H, Tsugawa K, et al. Sentinel node biopsy after neoadjuvant chemotherapy in cytologically proven node-positive breast cancer. Clin Breast Cancer.
2013;13:471-7.

36. Boughey JC, Suman VJ, Mittendorf EA, et al. Factors affecting sentinel lymph node identification rate after neoadjuvant chemotherapy for breast cancer patients enrolled in ACOSOG Z1071 (Alliance). Ann Surg 2015;261:547-52.
Cite this article as: Wang M, Wang M, Wang Z, Song Y, Gao P, Wang P, Wang C, Yu X, Wei F, Guo J, Xu Y. Nomogram for predicting axillary lymph node status after neoadjuvant chemotherapy in breast cancer. Transl Cancer Res 2020;9(11): 7054-7064. doi: 10.21037/tcr-20-2377 